\title{
A New Way to Slow Down Complex Molecules
}

\author{
A magnetic-field based method can slow molecular beams that cannot be \\ slowed using other techniques, unlocking the door to ultracold \\ polyatomic molecular physics.
}

By Mirco Siercke and Silke Ospelkaus

$\square$ tudying polyatomic molecules trapped at microkelvin temperatures promises to revolutionize precision metrology and to shed light on the fundamental quantum basis of chemistry. It could even lead to never-before-seen states of matter. To get the molecules

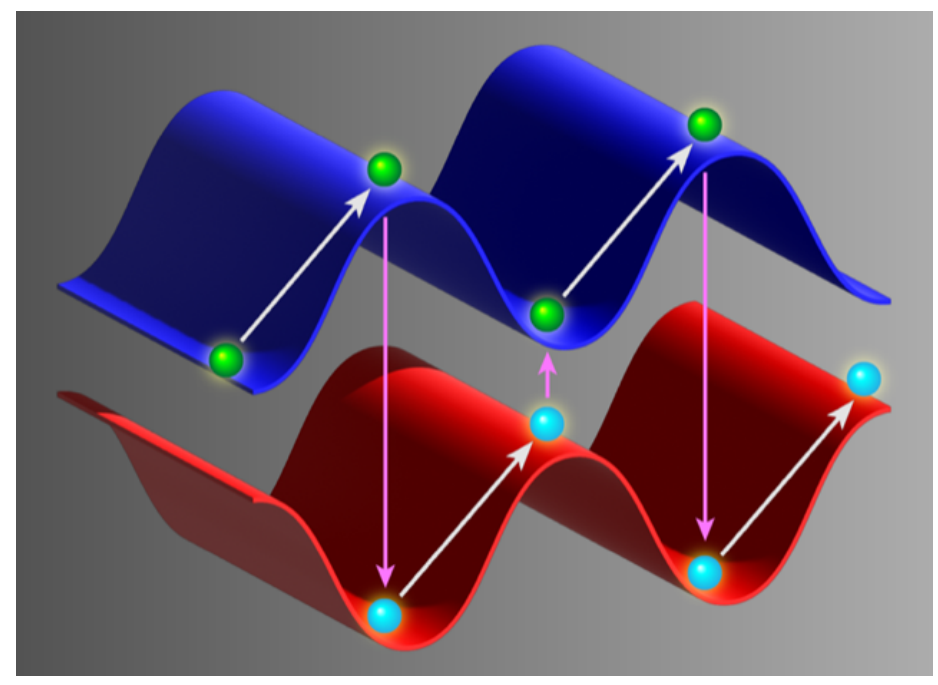

Figure 1: Fast-moving molecules in a "low-field-seeking" state (green circles) lose energy and slow down as they climb a magnetic-field gradient to a region of high field strength. When the molecules reach the top of a magnetic "hill," a laser induces a transition (pink arrows) to a "high-field-seeking" state (blue circles). Molecules in this state lose energy as they move down a magnetic-field gradient. By changing the state of the molecules before they can "roll down" the potential-energy gradient, they can be made to slow down continuously.

Credit: APS/Alan Stonebraker trapped, however, researchers first have to slow down a molecular beam nearly to a standstill. While such "trappable" velocities have been achieved for a few diatomic molecules using laser cooling, applying the technique to polyatomic molecules seems out of reach. Benjamin Augenbraun and colleagues, at Harvard University, have now shown that an alternative cooling technique called Zeeman-Sisyphus deceleration-first proposed by researchers at Imperial College London [1] - can be used instead [2]. The demonstration paves the way to trapping gases of ultracold polyatomic molecules and all the rich physics that comes with them.

Research in ultracold atomic physics has been incredibly fruitful over the last few decades, resulting in multiple Nobel Prizes and countless new applications. The comparatively new field of ultracold diatomic molecular physics promises an equally rich landscape of novel phenomena. So far, progress in this field has mainly been in the preparation of diatomic species at ultracold temperatures, which can be achieved either through the controlled association of atoms into molecules [3] or through the direct laser cooling to microkelvin temperatures of certain "ready-made" diatomic molecules [4-7].

Against the background of these advances, some groups have already set their eyes on polyatomic molecules, which, because of the additional degrees of freedom afforded by their rotational and vibrational states, promise an even greater leap in progress than their diatomic counterparts [8,9]. Certain polyatomic molecules, for example, have excitation modes that make them very sensitive to the electron's electric dipole moment, so trapping them could lead to the discovery of physics beyond the standard model. Furthermore, as molecules 
become more complex, the possibility opens up for studying novel ultracold chemistry and gaining an understanding of chirality in molecules. But while ultracold polyatomic molecules might offer greater experimental potential, they also come with greater experimental challenges.

Typically, optical techniques for cooling and slowing molecules get exponentially harder when more atoms are added to the molecule. Laser slowing any particles-whether atoms or molecules-requires thousands of photons to be scattered. While this is trivial for atoms because of their simple energy structures, it can only be achieved for a select handful of diatomic molecules. That's because molecules have extra degrees of freedom, which increase the number of the ways in which they can interact with photons. Some of these interactions can excite the molecules to "dark" vibrational or rotational energy sublevels that make further cooling impossible. Polyatomic molecules have even more of these dark states than diatomic molecules do, and they typically switch to a dark state after just a few photon interactions. Laser cooling is even more likely, therefore, to induce transitions that make polyatomic molecules experimentally inaccessible. As a result of this limitation, trapping and cooling of polyatomics has so far been restricted to molecules that can be decelerated using electric fields rather than photon scattering (see Viewpoint: Slowing Continuous Molecular Beams in a Rotating Spiral) [10]. The scheme demonstrated by Augenbraun and colleagues does not suffer from the limitations of laser cooling or electric-field-induced deceleration: It can be applied to any molecule that is paramagnetic, that is, molecules that have the inherent advantage of being magnetically trappable.

Zeeman-Sisyphus cooling relies on the fact that all paramagnetic molecules behave similarly in the "Paschen-Back" high-magnetic-field regime. In this regime, the molecules' ground-state structures have two energy levels: one with a low energy in high fields (high-field seeker) and one with a low energy in low fields (low-field seeker). Because of these states, a molecule in the low-field-seeking state loses energy as it "rolls up" a magnetic-field-strength gradient, while a high-field-seeking molecule loses energy as it "rolls down" this gradient. By using a laser to change them from the high-field-seeking state to the low-field-seeking state at the right times, molecules can be made to roll continuously up a potential-energy "hill" as they pass through regions of high and low magnetic-field strength, losing kinetic energy as they go (Fig. 1). This behavior likens the scheme to the famous myth of Sisyphus, who was condemned to roll a boulder up a steep hill over and over again.

The Harvard team's scheme differs from the original proposal for Zeeman-Sisyphus cooling [1] in a few key aspects [2]. Whereas the original Imperial group's scheme specified about a hundred permanent magnets to produce about a hundred magnetic hills and valleys (therefore requiring hundreds of transitions between the low-field-seeking and high-field-seeking states), the new scheme instead uses two sets of superconducting magnetic coils to implement just two hills. These hills are of such a height (2.8 T, corresponding to an energy loss of $1.9 \mathrm{~K}$ per ascent) that the molecules lose all their kinetic energy in just two stages. As such, while the original proposal requires the scattering of hundreds of photons from each molecule, the new experiment manages to bring the molecules to rest by scattering, on average, just seven photons each.

This low number of scattered photons is key to the significance of the new experiment. It allows Augenbraun and colleagues to circumvent the problem of polyatomic molecules decaying into random, experimentally inaccessible energy states after scattering just a handful of photons, making it possible to trap and investigate a plethora of paramagnetic polyatomic molecules in a new temperature regime and for much longer periods than ever before. The exact set of molecules that can be addressed by this scheme remains unknown, but the diversity of polyatomic molecules promises rich prospects for applications in physics and chemistry, with every species offering something new compared with others. Some of the first targets for investigation are likely to be barium monohydroxide and radium monohydroxide as these molecules are very similar to the calcium monohydroxide used in the new demonstration. These molecules are exciting, as their high mass makes them extremely sensitive probes of the electron's electric dipole moment, measurements of which could lead to beyond-standard-model physics.

Mirco Siercke: Leibniz University Hannover, Hanover, Germany Silke Ospelkaus: Leibniz University Hannover, Hanover, Germany 


\section{REFERENCES}

1. N. J. Fitch and M. R. Tarbutt, "Principles and design of a Zeeman-Sisyphus decelerator for molecular beams," ChemPhysChem 17, 3609 (2016).

2. B. L. Augenbraun et al., "Zeeman-Sisyphus deceleration of molecular beams," Phys. Rev. Lett. 127, 263002 (2021).

3. K.-K. Ni et al., "A high phase-space-density gas of polar molecules," Science 322, 231 (2008).

4. J. F. Barry et al., "Magneto-optical trapping of a diatomic molecule," Nature 512, 286 (2014).

5. L. W. Cheuk et al., " $\Lambda$-enhanced imaging of molecules in an optical trap," Phys. Rev. Lett. 121, 083201 (2018).
6. L. Caldwell et al., "Deep laser cooling and efficient magnetic compression of molecules," Phys. Rev. Lett. 123, 033202 (2019).

7. S. Ding et al., "Sub-doppler cooling and compressed trapping of YO molecules at $\mu \mathrm{K}$ temperatures," Phys. Rev. X 10, 021049 (2020).

8. M. Zeppenfeld et al., "Sisyphus cooling of electrically trapped polyatomic molecules," Nature 491, 570 (2012).

9. D. Mitra et al., "Direct laser cooling of a symmetric top molecule," Science 369, 1366 (2020).

10. S. Chervenkov et al., "Continuous centrifuge decelerator for polar molecules," Phys. Rev. Lett. 112, 013001 (2014). 